current was maintained, but the fast neurones adapted rapidly and did not, unlike the slow, return to firing after an excessive depolarization was reduced. This disparity is probably a result of greater sodium inactivation in the "fast" membrane.

When extracellular current-passing electrodes were used, only stimulation near the soma gave rise to repetitive discharges. The axonal diameter is smallest near the soma, with a membrane space constant $(\lambda)$ of about $0.6 \mathrm{~mm}$, so threshold is lowest at this point. For highest discharge frequencies it was necessary to depolarize the soma as well (either by stretching the receptor or with an intracellular electrode). Whatever the mixture of stimulation used, however, the shape of the action potential was constant, suggesting that it was indeed initiated at the soma-axonal junction. A small pre-potential was always visible; it could be dissociated from the main spike under sustained depolarization, remaining repetitive, but not leading to further spikes.

Under constant stretch, the slow receptors fired continuously, but the fast adapted rapidly, never firing for more than about $8 \mathrm{~s}$. In both, however, the generator potentials, which could be seen independently of action potentials when these were abolished by the administration of tetrodotoxin, were identical. This, of course, implies that the adaptation in fast receptors occurs after the production of the generator potential, in the spike-generating mechanism. This is quite surprising, for the geometries of the fast and slow dendrites are quite different and a priori might well have been thought to provide a basis for the different response properties. None the less, the two receptors seem to respond in essentially the same way until the spike generating mechanism is reached.

In contrast, Nishi and Sato (J. Physiol., 199, 383; 1968) confirm Hubbard's view that the connective tissue lamellae surrounding the unmyelinated axon terminal within a Pacinian corpuscle serve as a highpass filter, making rapid compression relatively effective in stimulating the terminal. Sustained compression of the bare terminal does indeed produce a sustained receptor (generator) potential, with a very slow decay, although this never occurs naturally, as the d.c. component of any compression is filtered out by the lamellae. However, the sign of the response depends on the direction of stimulation because the terminal is elliptical in cross-section. Compression along the short axis of the ellipse increases the terminal surface area and gives a depolarizing response, with a hyperpolarizing off-effect, while compression along the long axis does the opposite.

\section{THIN FILMS}

\section{Useful Sputtering}

\section{from a Correspondent}

Trrn films can be made by causing individual atoms to be ejected from a material by ionic bombardment. This technique, known as sputtering, is used extensively by the electronics industry for the deposition and etching of a wide range of materials. It now seems to have a new application in medical science, in the study of cytological material. This emerged at a conference on sputtering, organized by the Vacuum Group of the Institute of Physics and the Physical
Society in London on February 13. P. R. Stuart used a series of slides to compare the internal structure of normal and diseased blood cells after the ectoplasm had been removed by radio frequency ion etching. Using this technique, it is possible to examine the internal structure by scanning electron microscopy without recourse to the microtome for sectioning.

Many of the 150 people attending the conference showed great interest in another unusual application of radio frequency sputtering which was outlined by P. J. Harrop in a paper entitled "Sputtered PTFE". The properties of bulk PTFE, a material which has found many applications because of its inertness and low cocficient of friction, were compared with the properties of thin films $(0 \cdot 01-10$ microns $)$ of the polymer produced by diode radio frequency sputtering. The films adhered well to a wide range of materials and, although modified structurally by the deposition process, had very similar properties to the bulk material, in particular retaining their low coefficient of friction. Dielectric losses were found to be higher, partly perhaps because of a lack of cleanliness during deposition. Two differences which enhance the versatility of PTFE are the increased hardness of the sputtered material and its ability to withstand higher temperatures. These might make it more suitable for load bearing applications and extend its working range up to $400^{\circ} \mathrm{C}$. This latest development can be expected to bring a host of new electrical applications.

More conventional materials were reported by $\mathrm{P}$. R. Stuart in another paper, this time on stress measurements in sputtered deposits. These measurements are important for understanding thin film properties, which are known to depend on the internal stress in the material. Some very high stress values were recorded and the importance of the effect of the residual gases on stress in the films was demonstrated.

P. E. Bovey showed that the energy of the condensing atoms is another parameter which affects the properties of deposited films. Nucleation density of gold films was found to increase with an increase in the arrival energy of the atoms, resulting in a film composed of smaller crystallites, and with a higher resistivity and a lower temperature coefficient of resistance.

Substrate conditions during deposition in radio frequency sputtering systems were also discussed by G. N. Jackson, who has observed electron and ion bombardment of the substrate and preferential etching of the substrate adjacent to the edges of out-ofcontact masks.

Unlike the other contributors, C. Priestland was concerned with the performance of the non-grounded disk and annulus radio frequency sputtering systems. His investigations included total and partial pressure measurements, deposition rates and uniformity of deposit of different materials. The lively discussions revealed that disk and annulus systems were probably more electrically efficient and stable in the absence of magnets than simple disk and grounded electrode systems. But the latter give the best uniformity and are cheaper-preferences still vary.

There is now some doubt about the extent to which sputtered deposits adhere better than evaporated deposits. Mr Stuart reported evidence that metal films thicker than a few hundred ångströms adhere relatively poorly whether sputtered or evaporated unless the substrate is first cleaned by glow discharge. 